R. PEUCIENNIK

KODAI MATH. J.

10 (1987), $49-54$

\title{
REPRESENTATION OF ADDITIVE FUNCTIONALS ON MUSIELAK-ORLICZ SPACE OF VECTOR-VALUED FUNCTIONS
}

\author{
BY RYSZARD PŁUCIENNIK
}

In the paper [4] Hiai proved theorems on representation of additive functionals on vector-valued normed Köthe spaces. His theory, as is shown by the Example 2., does not contain an important and large class of non-solid Orlicz and Musielak-Orlicz spaces of vector-valued functions. Nevertheless, a very interesting idea of the proofs is so universal that it can be applied in the above case as well. It is necessary to change only proofs of Lemma 3.1 and Theorem 3.4 in which the following assumption

$$
\|f(t)\|_{X} \leqq\|g(t)\|_{X} \quad \text { for almost all } t \text { implies }\|f\|_{M} \leqq\|g\|_{M}
$$

is essential. Therefore, in order to avoid the repetition of argumentations presented in the paper [4], this note contains the modifications of Lemma 3.1 and Theorem 3.4. Then representation theorems for additive lower semicontinuous and continuous functionals are presented as a conclusion. Moreover, it is worth to notice that the representation theorem for bounded linear functionals, considered also by Hiai, in these spaces was elementarily proved in a particular case by Kozek (see [8]).

1. Introduction. Let $(T, \Sigma, \mu)$ be a positive, $\sigma$-finite and complete measure space. $\left(X,\|\cdot\|_{X}\right)$ denotes a separable real Banach space. iff

Definition 1. A function $M: X \times T \rightarrow[0, \infty]$ is said to be an $\mathscr{N}$-function,

a) $\quad M$ is $\mathscr{B} \times \Sigma$-measurable, where $\mathscr{B}$ denotes the $\sigma$-algebra of Borel subsets of $X$,

b) $M(\cdot, t)$ is even, convex, lower semicontinuous, continuous at zero and $M(0, t)=0$ for a. a. $t \in T$,

c) $\lim _{\|x\|_{X^{\rightarrow \infty}}} M(x, t)=\infty$ a.e. in $T$.

Let us assume that $\mathscr{N}$-function $M$ satisfies the so-called Condition $B$, which can be also formulated in the following simple form (see [15] Remark 1.5)

Received April 8, 1986 
B: For every natural numbers $n$ and $i$

$$
\int_{T_{n}} \sup _{\|x\|_{X}<i} M(x, t) d \mu<\infty,
$$

where $\left\{T_{n}\right\}$ is an increasing sequence of measurable sets such that $\mu\left(T_{n}\right)<\infty$ and $\bigcup_{n=1}^{\infty} T_{n}=T$.

In the following by $\mathscr{X}_{X}$ we will denote the set of all strongly measurable functions from $T$ into $X$. The famous Pettis theorem states that the strong measurability and the weak one are equivalent for separable Banach spaces. Therefore, we will say shortly "measurable function".

By Musielak-Orlicz space $L_{M}$ we mean the set of all functions $f \in \mathscr{X}_{X}$ for which there exists a constant $k>0$ such that

$$
I_{M}(k f)=\int_{T} M(k f(t), t) d \mu<\infty .
$$

The functional

$$
\|f\|_{M}=\inf \left\{a>0: I_{M}\left(a^{-1} f\right) \leqq 1\right\}
$$

is a norm in $L_{M}$. It is called the Luxemburg norm. If $M(x, t)=M(x, s)$ for every $t, s \in T$, then the space $L_{M}$ generated by $M$ is called an Orlicz space.

By $E_{M}$ we denote a subspace of finite elements, i.e.

$$
E_{M}=\left\{f \in \mathfrak{X}_{X}: I_{M}(k f)<\infty \text { for every } k>0\right\} .
$$

Obviously, $E_{M} \subset L_{M}$. The space $E_{M}$ equals the space of all $f \in L_{M}$ possessing absolutely continuous norms (see [14] Theorem 1.2. This theorem is also true without the assumption that the measure $\mu$ is non-atomic). Concerning properties of the space $E_{M}$ we refer to [8], [9] and [12] (Theorem 1.15).

Musielak-Orlicz spaces even Orlicz spaces, defined as above, are not normed Köthe spaces in general. The following natural example shows this fact.

Example 2. Let $X=R^{2}, T=(0,1), \Sigma$ be the $\sigma$-algebra of Lebesgue measurable subsets of $(0,1), \mu$ be the Lebesgue measure and define $M: R^{2} \rightarrow[0, \infty]$ by the formula

$$
M(r, s)=r^{2}+s^{4} .
$$

It is easy to verify that $M$ satisfies $\Delta_{2}$-condition (see [8] p. 268). Corollary 1.7.4 in [9] implies the equality $L_{M}=E_{M}$. Let $f_{1}$ and $f_{2}$ be two functions from $(0,1)$ into $R^{2}$ which are defined by the following formulas

We have

$$
\begin{array}{ll}
f_{1}(t)=\left(t^{-1 / 3}, t\right) & t \in(0,1) \\
f_{2}(t)=\left(t, t^{-1 / 4}\right) & t \in(0,1) .
\end{array}
$$

$$
\left\|f_{1}(t)\right\|_{R^{2}}=\sqrt{t^{-2 / 3}+t^{2}}>\sqrt{t^{2}+t^{-1 / 2}}=\left\|f_{2}(t)\right\|_{R^{2}}
$$


for every $t \in(0,1)$. On the other hand $I_{M}\left(f_{1}\right)=16 / 5$ and $I_{M}\left(f_{2}\right)=\infty$, therefore $f_{1} \in L_{M}$ and $f_{2} \notin L_{M}$ because $M$ satisfies $\Delta_{2}$-condition. Moreover, $\left\|f_{1}\right\|_{M}<\infty$ and $\left\|f_{2}\right\|_{M}=\infty$. Thus, the norm $\|\cdot\|_{M}$ in $L_{M}$ does not satisfy Condition (iv) in [4] p. 301 and the space $L_{M}$ cannot be solid.

2. Results. In this section, the most of notations are identical with those in Section 3 in [4]. We will present them for the convenience of reading.

Let $F: T \rightarrow 2^{X}$ be a multivalued function from $T$ into the family of subsets of the space $X$. Denote the sets

$$
D(F)=\{t \in T: F(t) \neq \varnothing\} \text { and } G(F)=\{(t, x) \in T \times X: x \in F(t)\}
$$

which are called the domain of $F$ and the graph of $F$, respectively. Also denote the inverse image of $F$ by

$$
F^{-1}(U)=\{t \in T: F(t) \cap U \neq \varnothing\}, \quad U \subset X .
$$

Since $(T, \Sigma, \mu)$ is complete, for $F: T \rightarrow 2^{X}$ such that $F(t)$ is closed for every $t \in T$, the following conditions are equivalent:

(1) $F^{-1}(C) \in \Sigma$ for every closed subset $C$ of $X$

(2) $F^{-1}(O) \in \Sigma$ for every open subset $O$ of $X$

(3) $D(F) \in \Sigma$ and there exists a sequence $\left\{f_{n}\right\}$ of measurable functions $f_{n}: D(F) \rightarrow X$ such that $F(t)=\operatorname{cl}\left\{f_{n}(t)\right\}$ for all $t \in D(F)$

(4) $G(F) \in \Sigma \times \mathscr{B}$.

A closed-valued function $F: T \rightarrow 2^{X}$ is called to be measurable if $F$ satisfies one of the above conditions. We will denote by $U[T, X]$ the collection of all measurable multivalued functions $F: T \rightarrow 2^{X}$ such that $F(t)$ is nonempty and closed for every $t \in T$.

Let $\mathcal{R}$ be a family of measurable functions in $\mathscr{X}_{X}$. The family $\mathscr{R}$ is said to be decomposable if $f \chi_{A}+g \chi_{T \backslash A} \in \mathscr{R}$ for each $f, g \in \mathscr{R}$ and $A \in \Sigma$, where $\chi_{A}$ denotes the characteristic function of a set $A$. Let $L_{1}$ denote the space of integrable functions from $T$ to the set of reals. Consider the space $E_{M} \times L_{1}$ with the norm $\|\cdot\|_{M}+\|\cdot\|_{1}$. If $F \in \mathcal{U}[T, X \times R]$ then the subset $S_{M, 1}(F)$ of $E_{M} \times L_{1}$ is defined by

$$
S_{M, 1}(F)=\left\{(f, \xi) \in E_{M} \times L_{1}:(f(t), \xi(t)) \in F(t) \text { a.e. }\right\} .
$$

The properties of subsets $S_{M, 1}(F)$ are presented in the following lemmas.

LEMMA 3. For every $F \in \mathcal{U}[T, X \times R]$ the set $S_{M, 1}(F)$ is closed in $E_{M} \times L_{1}$.

Proof. Let $\left\{\left(f_{n}, \xi_{n}\right)\right\}$ be a sequence in $S_{M, 1}(F)$ convergent to $(f, \xi) \in E_{M} \times L_{1}$. Let $\left\{\xi_{n_{k}}\right\}$ be a subsequence of $\left\{\xi_{n}\right\}$ which is convergent to $\xi a . e$. in $T$. In view of Remark $3^{\circ}$ in [7] the sequence $\left\{f_{n}\right\}$ is convergent in measure on each set $T_{\imath}(i=1,2, \cdots)$, where $T_{\imath}$ are from Condition $B$. Therefore, we can find a subsequence $\left\{f_{k, 1}\right\}$ of $\left\{f_{n_{k}}\right\}$ convergent to $f$ a.e. on $T_{1}$. Suppose that the 
sequence $\left\{f_{k, i}\right\}$ is convergent to $f$ a.e. on $T_{\imath}$. Since the sequence $\left\{f_{k, i}\right\}$ is convergent to $f$ in measure on the set $T_{\imath+1}$, then there exists a subsequence $\left\{f_{k, \imath+1}\right\}$ of $\left\{f_{k, i}\right\}$ such that $\left\{f_{k, \imath+1}\right\}$ is convergent to $f$ a.e. on $T_{\imath+1}$. Let us notice that so-called diagonal sequence $\left\{f_{k, k}\right\}$ is convergent to $f$ a.e. on $T$. Hence, by the closedness of the set $F(t)$ in the norm $\|\cdot\|_{X}+1 \cdot 1$, we obtain that $(f(t), \xi(t)) \in F(t)$ a.e. on $T$. Thus $(f, \xi) \in S_{M, 1}(F)$ and the lemma is proved.

LEMMA 4. If $F \in \mathcal{U}[T, X \times R]$ and $S_{M, 1}(F)$ is nonempty, then there exists a sequence $\left\{\left(f_{n}, \xi_{n}\right)\right\}$ in $S_{M, 1}(F)$ such that $F(t)=c l\left\{\left(f_{n}(t), \xi_{n}(t)\right)\right\}$ for all $t \in T$.

LEMMA 5. If $F \in \mathcal{U}[T, X \times R]$ and $S_{M, 1}(F)$ is nonempty and convex, then $F(t)$ is convex for a.e. $t \in T$.

If is easy to see that the proofs of these lemmas are the same as the proofs of lemmas 3.2 and 3.3 in [4].

Now, we give a theorem characterizing closed decomposable subsets in $E_{M} \times L_{1}$.

THEOREM 6. Let $\mathscr{R}$ be a nonempty subset of $E_{M} \times L_{1}$. Then $\mathcal{R}$ is closed and decomposable in $E_{M} \times L_{1}$ if and only if there exists a multivalued function $F \in \mathcal{U}[T, X \times R]$ such that $\mathcal{R}=S_{M, 1}(F)$.

Proof. Sufficiency is clear by Lemma 3.

The necessity will be proved in two steps.

I. Assume in addition that $\mathscr{R}$ contains $(0,0) . L_{1}(X)$ denotes the space of all Bochner integrable functions from $T$ into $X$. Define $\mathcal{R}_{1}=\mathscr{R} \cap\left(L_{1}(X) \times L_{1}\right)$ and $\mathscr{R}_{2}$ as the closure of $\mathcal{R}_{1}$ in $L_{1}(X) \times L_{1}$. Obviously, $\mathscr{R}_{2}$ is a nonempty, closed and decomposable subset of $L_{1}(X) \times L_{1}$. Applying Theorem 3.1 in [5] to the space $L_{1}(X) \times L_{1}=L_{1}(X \times R)$, where $X \times R$ is considered with the norm $\|(\cdot, \cdot)\|_{X \times R}=\|\cdot\|_{X}+|\cdot|$, we can find a multivalued function $F \in V[T, X \times R]$ such that

$$
\mathscr{R}_{2}=\left\{(f, \xi) \in L_{1}(X) \times L_{1}:(f(t), \xi(t)) \in F(t) \text { a.e. }\right\} .
$$

We will prove that $\mathscr{R}=S_{M, 1}(F)$. Put

$$
A_{n}=\left\{t \in T_{n}:\|f(t)\|_{X} \leqq n\right\} \quad n=1,2, \cdots,
$$

where $(f, \xi) \in E_{M} \times L_{1}$ and $T_{n}$ are from Condition $B$. Then $\left(f \chi_{A_{n}}, \xi \chi_{A_{n}}\right) \in L_{1}(X)$ $\times L_{1}$, and the fact $T \backslash A_{n} \downarrow \varnothing$ gives

$$
\left\|f \chi_{A_{n}}-f\right\|_{M}+\left\|\xi \chi_{A_{n}}-\xi\right\|_{1}=\left\|f \chi_{T \backslash A_{n}}\right\|_{M}+\left\|\xi \chi_{T \backslash A_{n}}\right\|_{1} \longrightarrow 0 \quad \text { as } n \rightarrow \infty,
$$

in view of the absolute continuity of norms $\|\cdot\|_{M}$ and $\|\cdot\|_{1}$. Hence, by $(0,0) \in \mathscr{R}$ we see that ' $\mathcal{R}_{1}$ is dense in $\mathscr{R}$ and $S_{M, 1}(F) \cap L_{1}(X \times R)=S_{M, 1}(F) \cap \mathscr{R}_{2}$ is dense in $S_{M, 1}(F)$. Since both $\mathscr{R}$ and $S_{M, 1}(F)$ are closed, it remains to show that $\mathscr{R}_{1} \subset$ $S_{M, 1}(F)$ and $S_{M, 1}(F) \cap \mathcal{R}_{2} \subset \mathcal{R}$. The first inclusion is obvious. To prove the second inclusion, we assume that $(f, \xi) \in S_{M, 1}(F) \cap \Omega_{2}$. Then there exists a sequence $\left\{\left(f_{k}, \xi_{k}\right)\right\}$ in $\mathscr{R}_{1}$ convergent to $(f, \xi)$ with respect to the norm in 
$L_{1}(X) \times L_{1}$. Hence, it can be assumed that $\left\|f_{k}(t)-f(t)\right\|_{X} \rightarrow 0$ a.e. Denote

$$
B_{n, k, i}=\left\{t \in T_{n}:\left\|f_{k}(t)-f(t)\right\|_{X}<i^{-1}\right\}
$$

for $n, k, i \geqq 1$, where $T_{n}$ are from Condition B. Obviously, for each fixed $n$ and $i$, we have $\mu\left(T_{n} \backslash B_{n, k, \imath}\right) \rightarrow 0$ as $k \rightarrow \infty$. For each fixed $n$, by Condition B there exists a $\delta>0$

$$
\int_{T_{n}\|X\|_{X} \leqq \delta} \sup _{1} M(x, t) d \mu \leqq 1
$$

For each $\eta>0$, let $i$ be such that $(i \eta)^{-1}<\delta$. Then

$$
\eta^{-1}\left\|f_{k}(t)-f(t)\right\|_{X}<(\imath \eta)^{-1}<\delta \quad \text { for } t \in B_{n, k, \imath} .
$$

Therefore we have

and so

$$
I_{M}\left(\eta^{-1}\left(f_{k}-f\right) \chi_{B_{n}, k, i}\right) \leqq 1
$$

$$
\left\|\left(f_{k}-f\right) \chi_{B_{n, k}, i}\right\|_{M} \leqq \eta \quad \text { for all } k .
$$

This fact and the absolute continuity of the norm of $f$ give

$$
\left\|f_{k} \chi_{B_{n, k, 2}}-f \chi_{T_{n}}\right\|_{M} \leqq\left\|\left(f_{k}-f\right) \chi_{B_{n, k}, i}\right\|_{M}+\left\|f \chi_{T_{n} \backslash B_{n, k, \imath}}\right\|_{M}<2 \eta
$$

for sufficiently large $k$. Since $\left(f_{k} \chi_{B_{n, k}, i}, \xi_{k} \chi_{B_{n}, k, i}\right) \in \mathscr{R}$ by $(0,0) \in \mathscr{R}$, it follows that $\left(f \chi_{T_{n}}, \xi \chi_{T_{n}}\right) \in \mathscr{R}$ for all $n$. Thus, $(f, \xi) \in \mathscr{R}$ and the proof of the equality $\mathscr{R}=S_{M, 1}(F)$ is finished.

II. If $(0,0) \notin \mathscr{R}$, then we use Hiai's argumentation (see [4] beginning of the proof of Theorem 3.4). This completes the proof.

We say that a functional $\Phi: E_{M} \rightarrow \bar{R}=[-\infty, \infty]$ is proper if $\Phi(f)>-\infty$ for all $f \in E_{M}$ and $\Phi \neq \equiv$. For a measurable function $f: T \rightarrow X$, let $\operatorname{Supp} f=$ $\{t \in T: f(t) \neq 0\}$. A functional $\Phi$ is called to be additive if $\Phi(f+g)=\Phi(f)+\Phi(g)$ for each $f, g \in E_{M}$ such that $\mu(\operatorname{Supp} f \cap \operatorname{Supp} g)=0$.

Also, Lemmas 4.1, 4.2, 4.3 and 4.4 in [4] are true in our case. The proofs of these lemmas are similar because they do not require directly the assumption that $L_{\rho}$ is solid. Now, using our modified lemmas and Hiai's method, we can prove the following representation theorems for additive lower semicontinuous and continuous functionals on Musielak-Orlicz spaces of vector-valued functions.

THEOREM 7. Let $\Phi: E_{M} \rightarrow \bar{R}$ be an additive lower semicontinuous proper functional. Then there exists a $\Sigma \times \mathscr{B}$-measurable function $\varphi: T \times X \rightarrow \bar{R}$ such that $\varphi(t, 0)=0$ a.e., $\varphi(t, \cdot)$ is lower semicontinuous and proper for every $t \in T$ and

$$
\Phi(f)=\int_{T} \varphi(t, f(t)) d \mu \quad \text { for every } f \in E_{M} .
$$

Moreover such a function $\varphi$ is unique up to sets of the form $N \times X$ with $\mu(N)=0$. 
THEOREM 8. If $\Phi: E_{M} \rightarrow R$ is an additive continuous functional, then there exists a function $\varphi: T \times X \rightarrow R$ such that $\varphi(t, 0)=0$ a.e., $\varphi$ satisfies Carathéodory conditions (i.e. $\varphi(t, x)$ is measurable in $t$ and continuous in $x)$ and

$$
\Phi(f)=\int_{T} \varphi(t, f(t)) d \mu \quad \text { for every } f \in E_{M} .
$$

Moreover such a function $\varphi$ is unique up to sets of the form $N \times X$ with $\mu(N)=0$.

\title{
REFERENCES
}

[1] L. Drewnowski and W. ORlicz, On orthogonally additive functionals, Bull. Acad. Polon. Sci., Sér. sci. math., astr. et phys., 16 (1968), 883-888.

[2] L. DREwnowski AND W. ORLICZ, On representation of orthogonally additive functionals, Bull. Acad. Polon. Sci., Sér. sci. math., astr. et phys., 17 (1969), 167-173.

[3] L. DrewnowsKi AND W. ORLICZ, Continuity and representation of orthogonally additive functionals, Bull. Acad. Polon. Sci., Sér. sci. math., astr. et phys., 17 (1969), 647-653.

[4] F. HIAI, Representation of additive functionals on vector-valued normed Köthe spaces, Kodai Math. J., 2 (1979), 300-313.

[5] F. Hial and H. UMEGAKI, Integrals, conditional expectations, and matrtingales of multivalued functions, J. Multivariate Anal., 7 (1977), 149-182.

[6] C. J. Himmelberg, Measurable relations, Fund. Math., 87 (1975), 53-72.

[7] H. Hudzik AND A. KamiŃska, Some remarks on convergence in Orlicz space, Comment. Math., 21.1 (1980), 81-88.

[8] A. KozeK, Orlicz spaces of functions with values in Banach spaces, Comment. Math., 19 (1977), 259-288.

[9] A. Kozek, Convex integral functionals on Orlicz spaces, Comment. Math., 21. 1 (1980), 109-135.

[10] J. Musielak, Orlicz spaces and modular spaces, Springer-Verlag Berlin Heidelberg New York Tokyo 1983 vol. 1034.

[11] J. Musielak and W. Orlicz, On modular spaces, Studia Math., 18 (1959), 49-65.

[12] R. PEUciennik, On some properties of the superposition operator in generalized Orlicz spaces of vector-valued functions, Comment. Math., 25.2 (1985), 321-337.

[13] R. PEUciennik, Boundedness of the superposition operator in generalized Orlicz spaces of vector-valued functions, Bull. Pol. Ac. : Math., 33 (1985), 531-540.

[14] R. Ptuciennik, Some remarks on compactness in Musielak-Orlicz space of vectorvalued functions, Fasciculi Math., 16 (1986), 11-17.

[15] M. Wista, Some remarks on the Kozek Condition (B), Bull. Pol. Ac. : Math., 32 (1984), 407-415.

\author{
Institute of Mathematics \\ TECHNICAL UNIVERSITY \\ Piotrowo $3 \mathrm{~A}$ \\ 60-965 Poznań, Poland
}

